\begin{tabular}{|c|c|}
\hline 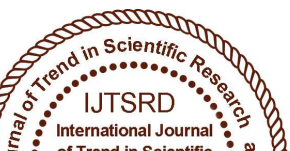 & $\begin{array}{l}\text { International Journal of Trend in Scientific } \\
\text { Research and Development (IJTSRD) }\end{array}$ \\
\hline 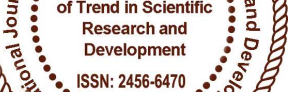 & International Open Access Journal \\
\hline 000 & ISSN No: 2456 - 6470 | www.ijtsrd.com | Volume - 2 | Issue - 3 \\
\hline
\end{tabular}

\title{
A Framework for Privacy-Enhancing Personalized Web Search
}

\author{
L. Sukanya ${ }^{1}$, R. Vijaya ${ }^{2}$ \\ ${ }^{1}$ MCA Final Year, ${ }^{2}$ Associate Professor \\ Department of MCA, Lakkireddy Balireddy College of Engineering, \\ Mylavaram, Andhra Pradesh, India
}

\begin{abstract}
The extending numerous of websites the all web customers are extended with the immense measure of data available in the web which is given by the Web Search Engine (WSE). The point of the WSE is to give the significant item to the client with the conduct of the client click were they performed. WSE give the pertinent outcome in the interest of the client visit click based strategy. From this technique no affirmation to the client protection and furthermore no securities were giving to their information. Consequently, clients were perplexed for their private data amid seek has turned into a noteworthy obstruction. They were numerous procedures were proposed by specialists the greater part of that considering the server side, it has given less security. For limiting the protection hazard here, we propose the user based system with the mix of Greedy strategy to keep the client information that we connected in Knowledge mining zone.
\end{abstract}

Keywords: Web Search Engine, user query logs, personalized search, content search and privacy preserving

\section{INTRODUCTION}

Web is one of the authority centres, which give the query thing to the customer with help of the WSE (Web search engine) [1]. It uses by securing information about various pages. WSE is a mechanical assembly which allows the web customer for finding information from the World Wide Web. WSE is the items that sweeps for and perceives the substance from the web engine or web database or web server with think about catchphrases or character

showed by the customer and finding particular goals on the World Wide Web [2]. Data chase and information recuperation on the Internet has discovered levels of prominence on web files. Various web lists like Google, Yahoo give an appropriate and unessential data to the customer in perspective of their chase. To keep up a vital separation from the inconsequential data the framework called Personalized Web Search (PWS) were rise. Inferring customer look for targets is basic in improving web searcher criticalness and redid look $[3,4]$. This depends upon the client profiles in context of the investigate $\log$ and the input session [5]. This data was produced using the unending inquiry asked for by the client, history of question, investigating, bookmarks et cetera.

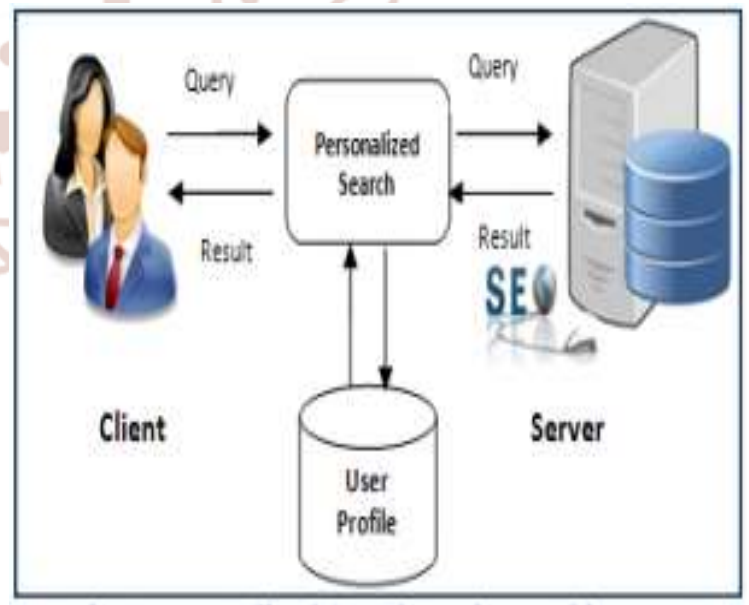

Fig 1: Personalized Search Engine Architecture

By these systems single data was sensibly revealing. While diverse web records manhandle information about people in like course, or with respect to 
particular get-togethers of people, altered scan for in setting of a customer profile is rise to the emerge person. Explores systems that re-attempt look happen exhibit their customers in different forms. The PWS gives a phenomenal way to cement and research work from mechanical labs on tweaking web look using customer logged scan for lead setting. It demonstrates a totally anonymized dataset, which has anonymized customer id, request in setting of the watchwords, terms of question, giving URLs and customer clicks. By utilizing these systems security of the client may be incident in context of tapping the related look for after, a sublime piece of the time go by objectives and giving their own specific data like their name, address, and so forth for this condition their protection may be spill.

It bears an imperative party of relationship to individuals, and a couple of these affiliations don't expect that data will be accumulated about a person to be adaptable. Those theories don't shield security issues moving from the nonattendance of affirmation for the client information. To giving better confirmation we propose a security protecting with the assistance of eager methodology by giving the mix technique for the coupling impact and keep the data catastrophe.

\section{Literature Review}

Author exhibited a decision theoretic structure/for redesiging shrewd information recuperation in setting of incredible customer demonstrate re-establishing, in which the framework reacts to each headway of the client by picking a structure activity to streamline an utility cutoff. Creator proposes [12] particular methodology to catch and endeavour two sorts of certain data:

(1) Perceiving related quickly going before question and using the request and the emerging outline things from pick fitting terms to broaden the present inquiry.

(2) Using these structures, creator develops a client side web scan for expert over a standard web crawler (Google) with no additional effort from the customer In [9] this paper, creator considers this issue and gives some preparatory conclusions. It shows an expansive scale appraisal structure for changed look considering question logs and after those reviews with the snap and profile-based frameworks. By isolating the outcomes, maker uncovers that changed sweep for has basic change over standard web looked on several demands, yet it has little impact on different request. Maker other than uncovers that both entire game-plan and unequivocally settings are central in invigorating analyzes execution for profile-based re-try search for systems. Maker tries to take a gander at whether personalization is constantly valuable under various conditions. The profile-based fix up examine for structures proposed in this paper isn't as persisting as the snap-based ones. They could enhance the intrigue accuracy on a couple of request; in any case they other than hurt particular demand. Since these systems are a long way from idealize, maker will proceed with his work to invigorate them in future [10]. It correspondingly finding for profile-based procedures, both entire game-plan and indisputably settings are basic in refreshing sweep for execution. The best blend of them can be more grounded than just utilizing both.

From the [13] author have investigated how to manhandle clear data, including demand history and research history inside a nearby request session, to invigorate data recovery execution. Utilizing the KLdivergence recovery show up as the introduction, maker proposed and considered four honest to goodness tongue models for setting unstable data recovery. The present work can be connected in two or three ways: First, it has beginning late analyzed some fabulously basic vernacular models for setting certain data. It is hypnotizing to grow more present day models to better undertaking demand history and inspect history. For instance, this may treat a clicked rundown diversely relying upon whether the present demand is a hypothesis or refinement of the past request. Second, the proposed models should be possible in any sensible structures. It at show develops a client side changed yield for master, which will weave a touch of the proposed estimations. Maker will in like path mean a customer thinks to audit sound judgment of these models in the considerable planned web look. Finally, maker ought to moreover consider a general recuperation framework for dynamic urgent improvement in careful information recuperation and think how to overhaul a touch of the parameters in the setting delicate recuperation models. In this paper [7] the creator studied the present hypothesis frameworks are inadequate in light of the way that they can't confirmation security assurance in all cases, and occasionally get awful data disappointment by performing superfluously theory. In this paper, producer proposes fix up shock, and builds up another theory structure that considers balanced security necessities. This structure satisfactorily keeps away from security impedance even in conditions where the 
present systems flop hopelessly, and achieves summed up tables that allow switch mean examination. This work [8] sets out a strong hypothetical establishment for making substitute hypothesis frameworks. For example, the insatiable calculation showed up in this paper isn't immaculate, as in it doesn't for the most part accomplish the smallest data disaster.

\section{Proposed System}

Without a doubt, confirmation concern is honest as far as possible in passing on true blue revamp search for applications, and how to achieve changed look however protecting clients' security. Here we proposed a customer side personalization which manage saving security and imagine conceivable future frameworks to absolutely ensure client affirmation. For protection, we acquaint our approach with digitalized media content in context of client profile data. For this, two focal techniques were conveyed: Automatic improvement of client profiles in context of our profile generator instrument and after that again suggestion structure considering the substance to checks the client enthusiasm for point of view of our customer side Meta information.

Above figure demonstrates our proposed outline which is works in the customer side part and here we shield the information from the server, so just we offer security to the customer client.

Every request from the customer client was given by the distinctive asking for to the server, this covers the proceeding on investigate logs or substance-based part, from this client can shield the information from the server.

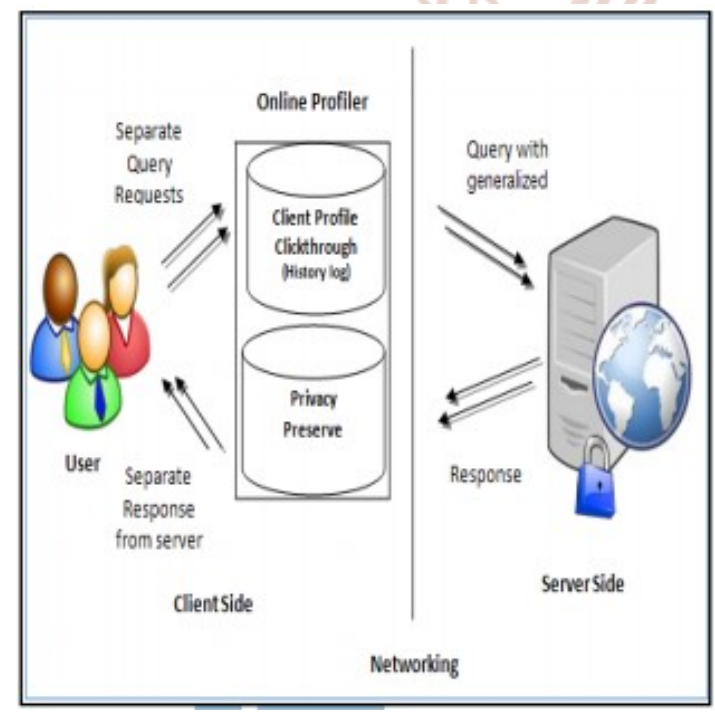

Fig 2: Proposed Architecture
In a comparable case our bit keeps up the online profiler about the customer along these lines it covers the snap logs and gives a challenge to the customer data. Starting now and into the not very hard to achieve, online profiler question was dealt with in the structure for speculation process, it is used to meet the basics to manage the customer profile and it relies upon the pre-dealing with the customer profiles. Game-plan, the client's demand execution and additionally their experience works out (e.g., saw beginning at now) and individual data could be joined with the client profile, considering structure of a for the most part wealthier client appears for personalization. The delicate germane data is for the most part not a standard perspective since it is totally secured and utilized on the customer side. A client's important data including client demand and snap logs history lives on the client's PC and is manhandled to better expect the client' data require and give a huge request things. Our proposed estimation utilizes the avaricious method in setting of the segregating force and data debacle insurance to secure the relations. Here it utilizes the spurred system to indicate up the request.

\section{Conclusion}

Web clients were growing an outcome of accessible of data from the web program considering the web look for instrument. With the developing number of client advantage motor must give the related thing considering their direct or in setting of the client execution. Giving related outcome to the client depends on their snap logs, ask for histories, bookmarks; by this security of the client may be occasion. For giving proper request by utilizing these systems the security of the client may misfortune. Most existing structure gives an enormous tangle to the private data amidst client look. Those methods don't secure protection issues and rising data hardship for the client information.

\section{References}

1) $\mathrm{Xu}$, Yabo, et al. "Privacy-enhancing personalized web search." Proceedings of the 16th international conference on World Wide Web. ACM, 2007

2) Xiao, Xiaokui, and Yufei Tao. "Personalized privacy preservation", Proceedings of the 2006 ACM SIGMOD international conference on Management of data. ACM, -2006. 
3) Shou, Lidan, et al. "Supporting Privacy Protection in Personalized Web Search." (2012): 1-1.

4) G. Chen, H. Bai, L. Shou, K. Chen, and Y. Gao, "Ups: Efficient Privacy Protection in Personalized Web Search," Proc. 34th Int'l ACM SIGIR Conf. Research and Development in Information, pp. 615- 624, 2011.

5) Dou, Zhicheng, Ruihua Song, and Ji-Rong Wen. "A large-scale evaluation and analysis of personalized search strategies", Proceedings of the 16th international conference on World Wide Web. ACM, 2007.

6) Shen, Xuehua, Bin Tan, and Cheng Xiang Zhai. "Implicit user modeling for personalized search." Proceedings of the 14th ACM international conference on Information and knowledge management. ACM, 2005.

7) T. Joachims, L. Granka, B. Pang, H. Hembrooke, and G. Gay, "Accurately Interpreting Clickthrough Data as Implicit Feedback," Proc. 28th Ann. Int'1 ACM SIGIR Conf. Research and Development in Information Retrieval (SIGIR '05), pp. 154-161, 2005.

8) Shen, Xuehua, Bin Tan, and Cheng Xiang Zhai. "Context-sensitive information retrieval using implicit feedback." Proceedings of the 28th annual international ACM SIGIR conference on Research and development in information retrieval. ACM, 2005.

9) $\mathrm{Xu}, \mathrm{Yabo}$, et al. "Online anonymity for personalized web services." Proceedings of the 18th ACM conference on Information and knowledge management. ACM, 2009.

10) A. Viejo and J. Castell_a-Roca, "Using Social Networks to Distort Users' Profiles Generated by
Web Search Engines," Computer Networks, vol. 54, no. 9, pp. 1343-1357, 2010.

11) K. Sugiyama, K. Hatano, and M. Yoshikawa, "Adaptive Web Search Based on User Profile Constructed without any Effort from Users," Proc. 13th Int'1 Conf. World Wide Web (WWW), 2004.

12) J. Teevan, S.T. Dumais, and E. Horvitz, "Personalizing Search via Automated Analysis of Interests and Activities," Proc. 28th Ann. Int'l ACM SIGIR Conf. Research and Development in Information Retrieval (SIGIR), pp. 449-456, 2005.

13) M. Spertta and S. Gach, "Personalizing Search Based on User Search Histories," Proc. IEEE/WIC/ACM Int'l Conf. Web Intelligence (WI), 2005.

14) Z. Dou, R. Song, and J.-R. Wen, "A Large-Scale Evaluation and Analysis of Personalized Search Strategies," Proc. Int'l Conf. World Wide Web (WWW), pp. 581-590, 2007.

15) X. Shen, B. Tan, and C. Zhai, "Context-Sensitive Information Retrieval Using Implicit Feedback," Proc. 28th Ann. Int'l ACM SIGIR Conf. Research and Development Information Retrieval (SIGIR), 2005.

16) $\mathrm{Y} . \mathrm{Xu}, \mathrm{K}$. Wang, B. Zhang, and Z. Chen, "Privacy-Enhancing Personalized Web Search," Proc. 16th Int'l Conf. World Wide Web (WWW), pp. 591-600, 2007.

17) X. Shen, B. Tan, and C. Zhai, "Privacy Protection in Personalized Search," SIGIR Forum, vol. 41, no. 1, pp. 4-17, 2007.

18) Y. Zhu, L. Xiong, and C. Verdery, "Anonymizing User Profiles for Personalized Web Search," Proc. 19th Int'l Conf. World Wide Web (WWW), pp. 1225-1226, 2010. 